\title{
Adipocyte DIO2 Expression Increases in Human Obesity but Is Not Related to Systemic Insulin Sensitivity
}

\author{
David Bradley (D), ${ }^{1}$ Joey Liu, ${ }^{1}$ Alecia Blaszczak, ${ }^{1}$ Valerie Wright, ${ }^{1}$ Anahita Jalilvand, ${ }^{1}$ \\ Bradley Needleman, ${ }^{2}$ Sabrena Noria, ${ }^{2}$ David Renton, ${ }^{2}$ and Willa Hsueh ${ }^{1}$ \\ ${ }^{1}$ Diabetes and Metabolism Research Center, Division of Endocrinology, Diabetes \& Metabolism, Department of Internal Medicine, \\ Wexner Medical Center, Ohio State University, Columbus, OH, USA \\ ${ }^{2}$ Center for Minimally Invasive Surgery, Department of General Surgery, Wexner Medical Center, Ohio State University, Columbus, \\ $\mathrm{OH} 43210$, USA
}

Correspondence should be addressed to David Bradley; david.bradley@osumc.edu

Received 14 March 2018; Accepted 25 June 2018; Published 15 July 2018

Academic Editor: Takayuki Masaki

Copyright (c) 2018 David Bradley et al. This is an open access article distributed under the Creative Commons Attribution License, which permits unrestricted use, distribution, and reproduction in any medium, provided the original work is properly cited.

\begin{abstract}
Deiodinase type II (D2), encoded by DIO2, catalyzes the conversion of T4 to bioactive T3. T3 not only stimulates adaptive thermogenesis but also affects adipose tissue (AT) lipid accumulation, mitochondrial function, inflammation, and potentially systemic metabolism. Although better defined in brown AT, the precise role of DIO2 expression in white AT remains largely unknown, with data derived only from whole fat. Therefore, the purpose of this study was to determine whether subcutaneous (SAT) and visceral (VAT) adipocyte-specific gene expression of DIO2 differs between obese and lean patients and whether these differences relate to alterations in mitochondrial function, fatty acid flux, inflammatory cytokines/adipokines, and ultimately insulin sensitivity. Accordingly, adipocytes of 73 obese and 21 lean subjects were isolated and subjected to gene expression analyses. Our results demonstrate that obese compared to lean human individuals have increased adipocyte-specific DIO2 expression in both SAT and VAT. Although higher DIO2 was strongly related to reduced fatty acid synthesis/oxidation and mitochondrial function, we found no relationship to proinflammatory cytokines or insulin resistance and no difference based on diabetic status. Our results suggest that adipocyte-derived DIO2 may play a role in weight maintenance but is likely not a major contributor to obesity-related insulin resistance.
\end{abstract}

\section{Introduction}

In spite of growing recognition, the obesity epidemic continues unabated in the United States (US). Over $2 / 3$ of the US adult population is now considered overweight or obese $[1,2]$. Obesity contributes to excess morbidity and mortality and adversely affects nearly every organ in the body [3]. With a growing appreciation for the negative health consequences, current treatment options for obesity and its complications remain insufficient and the underlying mechanisms behind obesity-induced complications have yet to be elucidated. Obesity fundamentally results when caloric intake exceeds total energy expenditure [4]. The role of nonshivering or adaptive thermogenesis (the generation of heat through uncoupling of mitochondrial respiration) in total energy expenditure is now being appreciated [5], with obese individuals demonstrating a reduced adaptive thermogenic capacity [6].

Deiodinase type II (D2) is encoded by the gene DIO2 and catalyzes the conversion of 3,5, $3^{\prime}, 5^{\prime}$-tetraiodothyronine (T4) to bioactive $3^{\prime}, 3^{\prime}, 5^{\prime}$-triiodothyronine (T3) by outer ring $5^{\prime}$-deiodination. Cold exposure increases circulating levels of $3^{\prime}, 3^{\prime}, 5^{\prime}$-triiodothyronine (T3) and stimulates catecholamineinduced adaptive thermogenesis $[6,7]$. Although the precise role of DIO2 expression and D2 activity in white adipose tissue (WAT) in contributing to the pathogenesis of human obesity and obesity-related comorbidities, such as type 2 diabetes 
(T2D), remains largely unknown, recent studies indicate a potential impact. Both polyphenols [8] and bile acids (BAs) [9] increase expression/activation of DIO2, improve mitochondrial respiration in BAT and skeletal muscle, and enhance weight loss. In addition, polymorphisms in DIO2 have been linked to increased insulin resistance, T2D risk, and poor glycemic control [10-12]. Thyroid hormone has major effects on lipid metabolism by activating hepatic and AT lipogenesis via induction of enzymes involved in fatty acid synthesis, including acetyl CoA carboxylase (ACC) and fatty acid synthase (FASN) [13], increasing fatty acid $\beta$-oxidation though carnitine palmitoyl transferase (CPT) $[14,15]$, and enhancing lipolysis in animal models [16]. Although gene expression of $\mathrm{DIO} 2$ in whole fat was recently found to be lower in AT of obese patients [17], adipocyte-specific expression and its relationship to mitochondrial function and fatty acid oxidation/synthesis in lean and obese human AT are not known.

Obesity is also associated with a state of low-grade chronic inflammation that contributes to varied comorbidities, including $\mathrm{T} 2 \mathrm{D}$ [3], and the balance between pro- and anti-inflammatory mediators and immune cells in AT maintains whole-body metabolism [18]. Thyroid hormone is a potent, but controversial, immunomodulatory factor [19-21], with gene targeting strategies in mice demonstrating an obligate role for thyroid hormone in immune cell development [22]. While an underlying proinflammatory state increases D2 activity and can affect lymphocyte proliferation [23], no human study to date has assessed the relationship between adipocyte DIO2 expression and pro- and anti-inflammatory factors and its possible impact on glucose homeostasis.

Therefore, the main purpose of this study was to test the hypothesis that SAT and VAT adipocyte gene expression of DIO2 differs between obese and lean human AT and that these differences will relate to (1) insulin resistance and (2) expression of genes involved in mitochondrial function, fatty acid flux, and inflammation.

\section{Materials and Methods}

2.1. Selection and Description of Participants. Ninetyfour consecutive eligible patients (BMI $18-40 \mathrm{~kg} / \mathrm{m}^{2}$; age $21-75 y$ ) who were scheduled to undergo elective abdominal surgery at the Center for Minimally Invasive Surgery or University Hospital East at the Ohio State University (OSU) Wexner Medical Center in Columbus, $\mathrm{OH}$, participated in this study. The study was approved by the OSU Institutional Review Board (IRB). All participants provided written informed consent and completed a comprehensive medical evaluation prior to enrollment. Potential participants who were current smokers, had evidence of end-stage renal or liver disease, had a history of prior organ transplantation, were on chronic pharmacologic steroid or anti-inflammatory use, and had a history of neoplastic disease or chemotherapy within the prior year, acquired immune deficiency syndrome, or $>10 \%$ body weight loss within 3 months of enrollment were excluded.
2.2. Study Design and Experimental Procedures. VAT biopsies were obtained from obese $(n=73$, age $44.8 \pm 11.9 \mathrm{y}$, BMI $\left.46.7 \pm 11.0 \mathrm{~kg} / \mathrm{m}^{2}\right)$ and lean $(n=21$, age $47.8 \pm 11.6 \mathrm{y}$, BMI $23.3 \pm 1.5 \mathrm{~kg} / \mathrm{m}^{2}$ ) patients during elective surgery, either Roux-en-Y gastric bypass or sleeve gastrectomy in obese subjects and elective cholecystectomy, hernia repair, Nissen fundoplication, or Heller procedure for achalasia in lean subjects. SAT biopsies were obtained from all the lean patients and a subset of the obese patients ( $n=35$, age $43.3 \pm 11.2 \mathrm{y}$, BMI $\left.48.0 \pm 8.5 \mathrm{~kg} / \mathrm{m}^{2}\right)$ (Table 1$)$. AT $(\sim 10$ grams) was obtained at surgery, rapidly transferred on ice, and then processed and fractionated within 15 minutes of obtaining the sample. Briefly, adipose for cell fractionation was minced, collagenase-digested, and fractionated into adipocytes and stromal vascular fractions (SVF) as previously described [24]. Adipocytes were immunodepleted using a CD45 antibody and then flash-frozen, and gene expression was determined using real-time quantitative polymerase chain reaction (qRT-PCR).

2.2.1. $q R T-P C R$. For $\mathrm{qRT}-\mathrm{PCR}$, adipose and adipocytes were analyzed for specific immune cell lineages (Cd3, Emr1) to assess T-cell and macrophage infiltration, respectively. Adipocyte RNA was analyzed for relative expression of DIO2, a gene associated with endocrine function (LEPTIN, ADI$P O Q)$, mitochondrial function (cell death activator (CIDEA), ATP synthase (ATP5A), and carnitine palmitoyltransferase $1 \mathrm{~B}(C P T 1 B)$ ), fatty acid $\beta$-oxidation (acetyl CoA dehydrogenase $(A C A D M))$, fatty acid synthesis (acetyl-CoA carboxylase (ACC2), fatty acid synthase (FASN), and diglyceride acyltransferase $(D G A T)$ ), adipocyte function (PPARgamma $(P P A R \gamma))$, innate immunity (NLRP3), and select proinflammatory or anti-inflammatory cytokines or chemoattractants (tumor necrosis factor alpha $(T N F \alpha)$, interleukin- $1 \beta$ $(I L-1 \beta)$, and plasminogen activator inhibitor-1 (PAI-1)). Adipocyte RNA was reverse-transcribed and amplified with TaqMan primer/probes (Life Technologies) or SYBR Green Primers (Sigma). RNA expression was normalized to PPIA. Gene expression values are shown as the fold change, defined by 2 -ddCT.

2.2.2. Analyses of Blood Samples. Plasma glucose, insulin, adiponectin, and leptin concentrations were measured by using enzyme-linked immunosorbent assays (Millipore, Billerica, MA).

\subsubsection{Calculations}

(1) Insulin Sensitivity and $\beta$-Cell Function. The homeostasis model assessment of insulin resistance (HOMA-IR) was used to determine the degree of insulin resistance in the subjects. The HOMA-IR accounts for fasting insulin levels relative to prevailing glucose levels and correlates well with more sensitive measures of insulin resistance such as the hyperinsulinemic-euglycemic clamp $[25,26]$. $\beta$-Cell function was determined by the HOMA- $\beta$, as previously described [26].

2.3. Statistical Analysis. Data were examined for normality according to the Shapiro-Wilk criteria and homogeneity of 
TABLE 1: Demographics and clinical characteristics of lean and obese patients.

\begin{tabular}{|c|c|c|c|}
\hline & $\begin{array}{l}\text { Lean subjects } \\
\quad(n=21)\end{array}$ & $\begin{array}{l}\text { Obese subjects with VAT biopsies } \\
\qquad(n=73)\end{array}$ & $\begin{array}{l}\text { Obese subjects with SAT biopsies } \\
\qquad(n=35)\end{array}$ \\
\hline Number of females/number of males (\% female) & $6 / 15(29)$ & $60 / 13(82)$ & $27 / 8(77)$ \\
\hline Age (years) & $47.8 \pm 11.6$ & $44.8 \pm 11.9$ & $43.3 \pm 11.2$ \\
\hline $\mathrm{BMI}\left(\mathrm{kg} / \mathrm{m}^{2}\right)$ & $23.3 \pm 1.5$ & $46.7 \pm 11.0^{*}$ & $48.0 \pm 8.5^{*}$ \\
\hline Fasting glucose (mg/dL) & $85.0 \pm 13.8$ & $93.4 \pm 22.3^{*}$ & $90.9 \pm 20.4^{*}$ \\
\hline Fasting insulin $(\mu \mathrm{IU} / \mathrm{mL})$ & $5.2 \pm 5.3$ & $18.8 \pm 16.8^{*}$ & $17.3 \pm 11.2^{*}$ \\
\hline HOMA-IR score & $1.1 \pm 1.0$ & $4.7 \pm 4.7^{*}$ & $4.1 \pm 3.1^{*}$ \\
\hline HOMA- $\beta$ score & $87.5 \pm 167.1$ & $236.0 \pm 193.8^{*}$ & $243.3 \pm 203.9$ \\
\hline Plasma adiponectin (ng/mL) & $11,294 \pm 8448$ & $6453 \pm 3723^{*}$ & $6380.3644^{*}$ \\
\hline Plasma leptin (ng/mL) & $26.9 \pm 11.3$ & $47.8 \pm 31.9^{*}$ & $55.4 \pm 40.7^{*}$ \\
\hline
\end{tabular}

Values are means \pm SD. BMI: body mass index; HOMA-IR: homeostasis model assessment of insulin resistance; HOMA- $\beta$ : homeostasis model assessment of beta-cell function. ${ }^{*}$ Value significantly different from the lean value $(p<0.05)$.

the variance by Levene's test. Groups were compared by one-way ANOVA for normally distributed variables and Mann-Whitney $U$ test for nonnormally distributed data. Pearson's correlation for variables with normal distribution and Spearman's correlation for variables with nonnormal distribution were calculated to assess association between variables. Multivariate linear regression analysis with independent variables of age, gender, and BMI was further calculated in a stepwise fashion. All data are presented as means \pm standard deviation unless otherwise noted.

\section{Results}

3.1. Characteristics of Patients. Demographic data, metabolic variables (fasting serum glucose, insulin, leptin, and adiponectin), and measures of insulin resistance and $\beta$-cell function are shown in Table 1. Overall, lean patients had lower BMI, fasting glucose, insulin and leptin levels, and HOMA-IR and HOMA-B scores. Lean patients had higher fasting serum adiponectin. There was no difference in age between the lean and obese patient groups.

3.2. Adipocyte Gene Expression of DIO2 Is Increased in Obese Human VAT and SAT but Is Not Related to Diabetic Status or Insulin Sensitivity. Although gene expression of whole fat DIO2 was recently found to be lower in VAT and SAT of obese patients [17] which contain different mixtures of adipocytes and a variety of immune cells, the expression of DIO2 specifically by the adipocyte is unknown. We thus determined the gene expression of DIO2 in human obese and lean SAT and VAT adipocytes by qRT-PCR. We observed a significant increase in adipocyte DIO2 gene expression in obese compared to lean SAT $(1.56 \pm 0.25$ versus $3.03 \pm 0.35 ; p=0.002)$ and VAT $(1.36 \pm 0.28$ versus $2.12 \pm 0.16 ; p<0.04$ ) (Figure $1(\mathrm{a})$ ). In addition, SAT DIO2 gene expression was directly associated with BMI (Figure 1(b)). As a significantly greater proportion of lean patients were male $(71 \%)$ and a greater proportion of obese patients were female $(82 \%$ in those undergoing VAT biopsies and $77 \%$ in those undergoing SAT biopsies), we compared VAT and SAT adipocyte DIO2 expression by gender. There was no difference between women and men in either visceral $(1.9 \pm 0.2$ versus $2.0 \pm 0.2, p=0.938)$ or subcutaneous adipocyte DIO2 expression (3.0 \pm 0.5 versus $2.3 \pm 0.5, p=0.270)$.

Polymorphisms in DIO2 have been linked to increased insulin resistance, T2D risk, and poor glycemic control [10-12], and T3 has been noted to have effects on mitochondrial function and energy expenditure, both of which can affect metabolic risk. However, we found no difference in adipocyte DIO2 gene expression between obese diabetic and nondiabetic patients in either SAT $(3.11 \pm 1.00$ versus $3.01 \pm 0.38, p=0.465)$ or VAT $(2.44 \pm 0.33$ versus $2.01 \pm$ $0.81, p=0.455$ ) (Figure $1(\mathrm{~d})$ ). In addition, neither SAT (Figure 1(c)) nor VAT (Figure 1(d)) DIO2 correlated with HOMA-IR, even after adjusting for BMI, age, and gender with multivariate linear regression (SAT: $p=0.701$; VAT: $p=0.797)$.

3.3. Adipocyte Gene Expression of DIO2 Is Related to Fatty Acid Synthesis/Beta-Oxidation and Mitochondrial Function. We next determined whether adipocyte DIO2 expression is related to expression of genes involved in fatty acid flux and mitochondrial function (Table 2). Overall adipocyte VAT and SAT DIO2 gene expression was inversely related to markers of mitochondrial function (CIDEA, ATP5A, PGC1 $\alpha$, and $C P T 1 B)$, fatty acid oxidation $(A C A D M)$, and fatty acid synthesis (ACC2, FASN, and DGAT). DIO2 expression was inversely related to the anti-inflammatory genes $A D I P O Q$ and PPARgamma in both VAT and SAT, but of the proinflammatory genes, only $P A I-1$ had a significant relationship to DIO2 expression. There were no significant correlations with LEPTIN, TNF $\alpha, N L R P 3$, or $I L-1 \beta$.

\section{Discussion and Conclusions}

The main purpose of this study was to test the hypothesis that SAT and VAT adipocyte gene expression of DIO2 differs between obese and lean human AT and that these differences relate to (1) markers of mitochondrial function and fatty acid flux, (2) systemic insulin resistance, and (3) pro- and antiinflammatory adipokines/cytokines. Our data demonstrates 


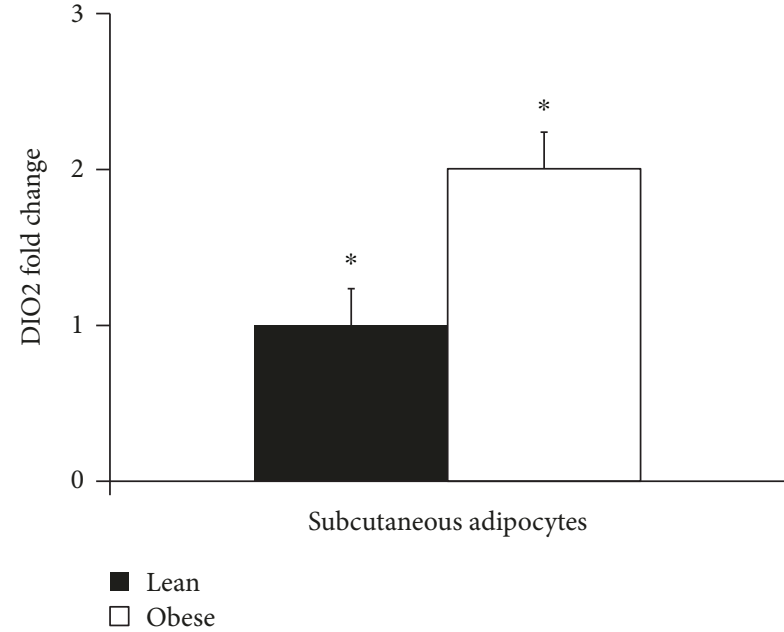

(a)

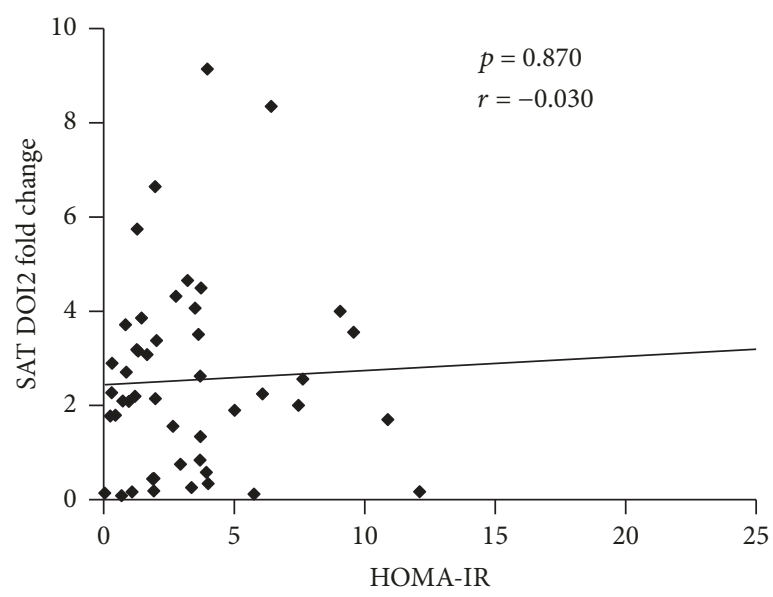

(c)

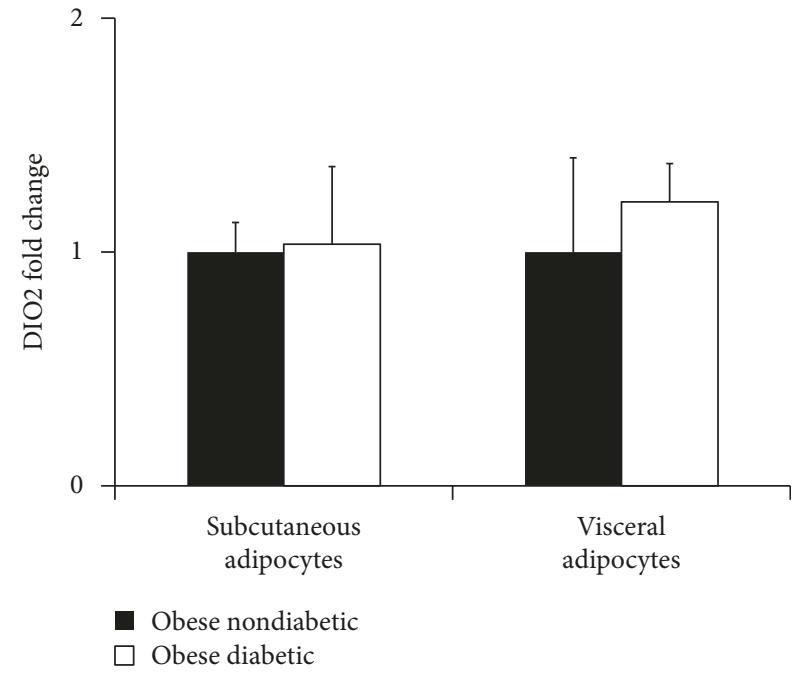

(b)

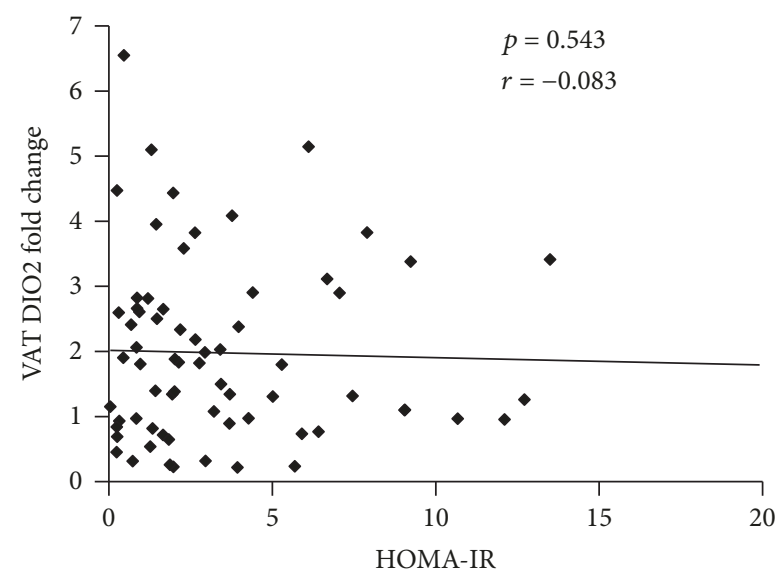

(d)

Figure 1: (a) Adipocyte expression of DIO2 in subcutaneous (SAT) and visceral (VAT) adipose tissue of lean and obese subjects and (b) obese diabetic and obese nondiabetic subjects analyzed by one-way ANOVA. Data presented as mean \pm SEM. ${ }^{*} p<0.04$. Correlation analyses (Pearson/Spearman) between adipocyte gene expression of DIO2 in SAT and VAT with HOMA-IR (c, d). HOMA-IR values used for correlation analyses were available for a subset of patients ( $n=16$ lean and $n=56$ obese).

that obese individuals exhibit increased adipocyte $\mathrm{DIO} 2$ expression compared to lean individuals in both SAT and VAT. There were strong relationships between higher DIO2 and reduced fatty acid synthesis/oxidation and mitochondrial function and adiponectin and $\operatorname{PPAR} \gamma$, and there was no relationship to inflammatory markers except for PAI-1. The clear lack of an association with insulin resistance and the finding of no difference in gene expression between obese diabetic and nondiabetic subjects suggest that adipocyte-derived $\mathrm{DIO} 2$ is not a major contributor to glucose homeostasis.

An imbalance between caloric intake and energy expenditure results in weight gain and ultimately over time in obesity [4]. Although traditionally energy expenditure has been defined as the summation of energy expended through physical activity, resting or basal metabolic rate, and nutrient breakdown, a more prominent role for nonshivering or adaptive thermogenesis is now being appreciated.
Adaptive thermogenesis is a component of energy expenditure whereby mammals generate heat through the uncoupling of mitochondrial respiration in brown adipose tissue (BAT) [5]. An expansive body of literature now highlights the role of adaptive thermogenesis and reduced thermogenic capacity in human obesity [6]. However, while the contribution of BAT to adaptive thermogenesis is well studied, the "beiging" of adipocytes within white adipose tissue (WAT) also appears to be an important component to adaptive thermogenesis. Beige adipocytes are induced by cold and/ or catecholamine-induced adrenergic stimulation and lead to augmented lipolysis of AT, increased adaptive thermogenesis, and higher total energy expenditure [27]. Cold exposure also increases the levels of biologically active T3, which enhances mitochondrial function and amplifies catecholamine-induced adaptive thermogenesis $[6,7]$.

Type II iodothyronine deiodinase (D2) is encoded by the gene $\mathrm{DIO} 2$ whose major function is to enzymatically convert 
TABle 2: Correlation coefficients $(r)$ between adipocyte DIO2 gene expression (fold change) in visceral (VAT) and subcutaneous (SAT) adipose tissue and gene expression of markers of mitochondrial function, fatty acid oxidation/synthesis, and proand anti-inflammatory genes.

\begin{tabular}{|c|c|c|}
\hline & $\begin{array}{c}\text { VAT DIO2 } \\
\text { gene expression } \\
(n=21 \text { lean; } n=73 \text { obese })\end{array}$ & $\begin{array}{c}\text { SAT DIO2 } \\
\text { gene expression } \\
(n=21 \text { lean; } n=35 \text { obese })\end{array}$ \\
\hline \multicolumn{3}{|c|}{ Mitochondrial function genes } \\
\hline CIDEA & $r=-0.365^{*}$ & $r=-0.423^{*}$ \\
\hline ATP5A & $r=-0.612^{*}$ & $r=-0.601^{*}$ \\
\hline PGC1 $\alpha$ & $r=-0.493^{*}$ & - \\
\hline CPT1B & $r=-0.436^{*}$ & $r=-0.483^{*}$ \\
\hline \multicolumn{3}{|c|}{ Fatty acid beta-oxidation genes } \\
\hline ACADM & $r=-0.586^{*}$ & $r=-0.549^{*}$ \\
\hline \multicolumn{3}{|c|}{ Fatty acid synthesis genes } \\
\hline ACC2 & $r=-0.460^{*}$ & $r=-0.510^{*}$ \\
\hline FASN & $r=-0.259$ & $r=-0.481^{*}$ \\
\hline DGAT & $r=-0.441^{*}$ & $r=-0.411^{*}$ \\
\hline \multicolumn{3}{|c|}{ Proinflammatory mediator of gene expression } \\
\hline IL-1 $\beta$ & $r=-0.091$ & $r=+0.045$ \\
\hline Leptin & $r=-0.115$ & $r=-0.260$ \\
\hline NLRP3 & $r=-0.126$ & $r=+0.026$ \\
\hline PAI-1 & $r=+0.602^{*}$ & $r=+0.324^{*}$ \\
\hline $\mathrm{TNF} \alpha$ & $r=-0.095$ & $r=+0.149$ \\
\hline \multicolumn{3}{|c|}{ Anti-inflammatory Mediator of gene expression } \\
\hline $\operatorname{PPAR} \gamma$ & $r=-0.656^{*}$ & $r=-0.562^{*}$ \\
\hline ADIPOQ & $r=-0.581^{*}$ & $r=-0.485^{*}$ \\
\hline
\end{tabular}

ADIPOQ: adiponectin; CIDEA: cell death activator; ATP5A: ATP synthase 5A; CPT1B: carnitine palmitoyltransferase 1B; ACADM: acetyl CoA dehydrogenase; ACC2: acetyl CoA carboxylase; FASN: fatty acid synthase; DGAT: diglyceride acyltransferase; PPAR $\gamma$ : PPARgamma; TNF $\alpha$ : tumor necrosis factor alpha; IL: interleukin; PAI-1: plasminogen activator inhibitor-1. Innate immunity (NLRP3). ${ }^{*} p<0.05$.

thyronine (T4) to T3 [28]. In healthy human subjects, 70\% of the extrathyroidal production of T3 is mediated by D2 [29]. Although D2 is expressed in a large number of cell types including chondrocytes and osteoblasts [30], cochlear and retinal cells [31], and tanycytes in the walls of the third ventricle of the brain [32], among many others [9], the potential role of AT-derived D2 in metabolic disease is unclear. Dietary supplementation with the bile acid cholic acid in mice reverses HFD-induced weight gain by activating D2 and converting T4 to T3 through TGR5 receptor activation. This activation results in increased expression of factors involved in mitochondrial biogenesis, oxidative phosphorylation (PGC- $1 \alpha$, CPT-1, UCP-1, and UCP-3), and fatty acid lipolysis [33], which subsequently improve systemic metabolism in murine models. In fact, the metabolic effects of BAs are attenuated in $\mathrm{DIO} 2^{-1-}$ mice [9]. Although gene expression of DIO2 in whole fat was reported to be lower in VAT and SAT of obese patients [17], the expression by adipocytes and the relationship between $\mathrm{DIO}$, mitochondrial function, and fatty acid oxidation/synthesis in lean and obese AT remain unknown.

In contrast to that observed in whole fat [17], we now report that DIO2 expression in isolated adipocytes is actually higher in human obesity and that increased DIO2 is related to lower mitochondrial gene expression and lipid oxidation, potentially consistent with its strong correlation with BMI in SAT. However, we found no relationship with systemic insulin resistance, suggesting that $\mathrm{DIO} 2$ from the adipocyte likely has minimal effects on human insulin sensitivity and glucose metabolism. In mature adipocytes, mitochondrial dysfunction reduces fatty acid oxidation [34] and promotes lipid accumulation [35]. In a state of chronic nutrient excess, as seen in obesity, these sequelae occur through the overproduction of reactive oxygen species (ROS). Both HFD feeding and hyperglycemia increase ROS production in mouse adipocytes [36,37], and oxidative stress is amplified in obese human subjects [38]. In turn, excess fatty acids further reduce mitochondrial biogenesis and gene expression, increase ROS, and lead to insulin resistance [34]. In our obese subjects, the strong inverse relationship between adipocyte $\mathrm{DIO} 2$ and key markers of mitochondrial function and fatty acid oxidation, coupled with the known effects of thyroid hormone to increase fatty acid $\beta$-oxidation $[14,15]$, could indicate a compensatory, but inadequate, response by the adipocyte to restore mitochondrial function and limit harmful lipid accumulation. Future studies, however, are warranted to evaluate these potentially important relationships.

A state of low-grade chronic inflammation is also present in obesity and leads to numerous comorbidities, including T2D [3]. In fact, TNF $\alpha$-mediated ROS accumulation leads to insulin resistance in preadipocytes [39]. Chronic inflammation has also been shown to increase D2 activity. In the current study, we noted negative relationships between DIO2 and anti-inflammatory gene expression of PPAR $\gamma$ and $A D I P O Q$ and no association with well-known proinflammatory mediators of insulin sensitivity, including $I L-1 \beta, T N F \alpha$, leptin, and NLRP3. These findings may explain the lack of an association between $\mathrm{DIO} 2$ and insulin resistance and suggest that $\mathrm{DIO} 2$ is not a major driver behind adipocyte-mediated proinflammatory activity and reduced insulin sensitivity.

Our study has several important limitations. Many of our findings are purely associative and may not implicitly constitute a cause-and-effect relationship. In addition, the lack of an association with $\mathrm{DIO} 2$ and insulin sensitivity could be due to insufficient power or subject sample size. However, the clear lack of a correlation in a substantial number of lean and obese human subjects in both SAT and VAT makes a meaningful relationship unlikely. We also measured DIO2 expression exclusively in AT adipocytes and not specifically in beiging cells, a significant factor in thermogenesis [40] which impacts weight maintenance. Despite no significant difference in age, our lean participants were predominantly male, while the obese subjects were largely female. A comparison of DIO2 expression in both VAT and SAT was similar in male compared to female subjects. In addition, after multivariate adjustment for gender, our findings still consistently 
failed to demonstrate an important relationship between DIO2 and IR or DIO2 and inflammation.

In conclusion, our findings indicate that $\mathrm{DIO} 2$ expression is upregulated in obese subcutaneous and visceral human adipocytes. However, in spite of significant relationships between $\mathrm{DIO} 2$ and reduced mitochondrial function, fatty acid oxidation, and anti-inflammatory adipokines, there was no association with systemic insulin resistance and no difference in diabetic compared to nondiabetic obese human subjects. Our results thus suggest that human adipocyte DIO2 is strongly related to body weight but does not play a major role in overall glucose homeostasis.

\section{Data Availability}

All data related to published results can be supplied upon request to the corresponding author.

\section{Disclosure}

David Bradley and Willa Hsueh had full access to all the data in the study and take responsibility for the integrity of the data and the accuracy of the data analysis.

\section{Conflicts of Interest}

The authors declare that they have no conflicts of interest.

\section{Authors' Contributions}

David Bradley designed the study, assisted in performing the experiments, collated and analyzed the data, and wrote the manuscript. Joey Liu assisted in performing the experiments and reviewed/edited the manuscript. Alecia Blaszczak and Anahita Jalilvand assisted in performing the experiments and reviewed/edited the manuscript. Valerie Wright assisted in performing the experiments. Bradley Needleman performed the surgeries and reviewed/edited the manuscript. Sabrena Noria performed the surgeries and reviewed/edited the manuscript. David Renton performed the surgeries and reviewed/edited the manuscript. Willa Hsueh designed the study, obtained funding, reviewed the data, contributed to the discussion, and reviewed/edited the manuscript.

\section{Acknowledgments}

The authors thank Andrew Suzo and Kayla Diaz for the help with the subject recruitment, Guy Brock and David Kline for the statistical support, and the study subjects for their participation. This study was supported by grants from the American Diabetes Association (1-16-ICTS-049) and the National Institutes of Health KL2 Scholar Award (KL2TR001068).

\section{References}

[1] C. L. Ogden, M. D. Carroll, B. K. Kit, and K. M. Flegal, "Prevalence of childhood and adult obesity in the United States, 2011-2012," JAMA, vol. 311, no. 8, pp. 806-814, 2014.
[2] Z. J. Ward, M. W. Long, S. C. Resch et al., "Redrawing the US obesity landscape: bias-corrected estimates of state-specific adult obesity prevalence," PLoS One, vol. 11, no. 3, article e0150735, 2016.

[3] K. B. Schelbert, "Comorbidities of obesity," Primary Care, vol. 36, no. 2, pp. 271-285, 2009.

[4] S. J. Guyenet and M. W. Schwartz, "Regulation of food intake, energy balance, and body fat mass: implications for the pathogenesis and treatment of obesity," The Journal of Clinical Endocrinology \& Metabolism, vol. 97, no. 3, pp. 745-755, 2012.

[5] J. E. Silva, "Physiological importance and control of nonshivering facultative thermogenesis," Frontiers in Bioscience, vol. S3, no. 1, pp. 352-371, 2011.

[6] J. E. Silva, "Thermogenic mechanisms and their hormonal regulation," Physiological Reviews, vol. 86, no. 2, pp. 435-464, 2006.

[7] J. E. Silva, "The thermogenic effect of thyroid hormone and its clinical implications," Annals of Internal Medicine, vol. 139, no. 3, pp. 205-213, 2003.

[8] W. S. da-Silva, J. W. Harney, B. W. Kim et al., "The small polyphenolic molecule kaempferol increases cellular energy expenditure and thyroid hormone activation," Diabetes, vol. 56, no. 3, pp. 767-776, 2007.

[9] M. Watanabe, S. M. Houten, C. Mataki et al., "Bile acids induce energy expenditure by promoting intracellular thyroid hormone activation," Nature, vol. 439, no. 7075, pp. 484-489, 2006.

[10] L. H. Canani, C. Capp, J. M. Dora et al., "The type 2 deiodinase A/G (Thr92Ala) polymorphism is associated with decreased enzyme velocity and increased insulin resistance in patients with type 2 diabetes mellitus," The Journal of Clinical Endocrinology \& Metabolism, vol. 90, no. 6, pp. 3472-3478, 2005.

[11] J. M. Dora, W. E. Machado, J. Rheinheimer, D. Crispim, and A. L. Maia, "Association of the type 2 deiodinase Thr92Ala polymorphism with type 2 diabetes: case-control study and meta-analysis," European Journal of Endocrinology, vol. 163, no. 3, pp. 427-434, 2010.

[12] X. Zhang, J. Sun, W. Han et al., "The type 2 deiodinase Thr92Ala polymorphism is associated with worse glycemic control in patients with type 2 diabetes mellitus: a systematic review and meta-analysis," Journal of Diabetes Research, vol. 2016, Article ID 5928726, 6 pages, 2016.

[13] B. Blennemann, P. Leahy, T. S. Kim, and H. C. Freake, "Tissuespecific regulation of lipogenic mRNAs by thyroid hormone," Molecular and Cellular Endocrinology, vol. 110, no. 1-2, pp. 1-8, 1995.

[14] F. L. Hoch, "Lipids and thyroid hormones," Progress in Lipid Research, vol. 27, no. 3, pp. 199-270, 1988.

[15] J. A. Stakkestad and J. Bremer, "The outer carnitine palmitoyltransferase and regulation of fatty acid metabolism in rat liver in different thyroid states," Biochimica et Biophysica Acta (BBA) - Lipids and Lipid Metabolism, vol. 750, no. 2, pp. 244-252, 1983.

[16] P. Arner, A. Wennlund, and J. Ostman, "Regulation of lipolysis by human adipose tissue in hyperthyroidism," The Journal of Clinical Endocrinology and Metabolism, vol. 48, no. 3, pp. 415-419, 1979.

[17] A. Kurylowicz, M. Jonas, W. Lisik et al., "Obesity is associated with a decrease in expression but not with the hypermethylation of thermogenesis-related genes in adipose tissues," Journal of Translational Medicine, vol. 13, no. 1, p. 31, 2015. 
[18] J. R. Brestoff and D. Artis, "Immune regulation of metabolic homeostasis in health and disease," Cell, vol. 161, no. 1, pp. 146-160, 2015.

[19] K. Dorshkind and N. D. Horseman, "The roles of prolactin, growth hormone, insulin-like growth factor-I, and thyroid hormones in lymphocyte development and function: insights from genetic models of hormone and hormone receptor deficiency," Endocrine Reviews, vol. 21, no. 3, pp. 292-312, 2000.

[20] G. D. Snell, "Dwarf, a new Mendelian recessive character of the house mouse," Proceedings of the National Academy of Sciences of the United States of America, vol. 15, no. 9, pp. 733-734, 1929.

[21] K. Dorshkind and N. D. Horseman, "Anterior pituitary hormones, stress, and immune system homeostasis," BioEssays, vol. 23, no. 3, pp. 288-294, 2001.

[22] C. Arpin, M. Pihlgren, A. Fraichard et al., "Effects of $\mathrm{T} 3 \mathrm{R} \alpha 1$ and $\mathrm{T} 3 \mathrm{R} \alpha 2$ gene deletion on $\mathrm{T}$ and $\mathrm{B}$ lymphocyte development," The Journal of Immunology, vol. 164, no. 1, pp. 152-160, 2000.

[23] K. W. Kelley, D. A. Weigent, and R. Kooijman, "Protein hormones and immunity," Brain, Behavior, and Immunity, vol. 21, no. 4, pp. 384-392, 2007.

[24] C. M. Halleux, P. J. Declerck, S. L. Tran, R. Detry, and S. M. Brichard, "Hormonal control of plasminogen activator inhibitor-1 gene expression and production in human adipose tissue: stimulation by glucocorticoids and inhibition by catecholamines," The Journal of Clinical Endocrinology \& Metabolism, vol. 84, no. 11, pp. 4097-4105, 1999.

[25] R. Muniyappa, S. Lee, H. Chen, and M. J. Quon, "Current approaches for assessing insulin sensitivity and resistance in vivo: advantages, limitations, and appropriate usage," American Journal of Physiology-Endocrinology and Metabolism, vol. 294, no. 1, pp. E15-E26, 2008.

[26] D. R. Matthews, J. P. Hosker, A. S. Rudenski, B. A. Naylor, D. F. Treacher, and R. C. Turner, "Homeostasis model assessment: insulin resistance and beta-cell function from fasting plasma glucose and insulin concentrations in man," Diabetologia, vol. 28, no. 7, pp. 412-419, 1985.

[27] S. Collins and R. S. Surwit, "The $\beta$-adrenergic receptors and the control of adipose tissue metabolism and thermogenesis," Recent Progress in Hormone Research, vol. 56, no. 1, pp. 309328, 2001.

[28] R. A. e Drigo and A. C. Bianco, "Type 2 deiodinase at the crossroads of thyroid hormone action," The International Journal of Biochemistry \& Cell Biology, vol. 43, no. 10, pp. 1432-1441, 2011.

[29] A. C. Bianco, D. Salvatore, B. Gereben, M. J. Berry, and P. R. Larsen, "Biochemistry, cellular and molecular biology, and physiological roles of the iodothyronine selenodeiodinases," Endocrine Reviews, vol. 23, no. 1, pp. 38-89, 2002.

[30] M. Miura, K. Tanaka, Y. Komatsu et al., "Thyroid hormones promote chondrocyte differentiation in mouse ATDC5 cells and stimulate endochondral ossification in fetal mouse tibias through iodothyronine deiodinases in the growth plate," Journal of Bone and Mineral Research, vol. 17, no. 3, pp. 443-454, 2002.

[31] L. Ng, R. J. Goodyear, C. A. Woods et al., "Hearing loss and retarded cochlear development in mice lacking type 2 iodothyronine deiodinase," Proceedings of the National Academy of Sciences of the United States of America, vol. 101, no. 10, pp. 3474-3479, 2004.
[32] B. C. G. Freitas, B. Gereben, M. Castillo et al., "Paracrine signaling by glial cell-derived triiodothyronine activates neuronal gene expression in the rodent brain and human cells," The Journal of Clinical Investigation, vol. 120, no. 6, pp. 2206-2217, 2010.

[33] L. A. de Jesus, S. D. Carvalho, M. O. Ribeiro et al., "The type 2 iodothyronine deiodinase is essential for adaptive thermogenesis in brown adipose tissue," The Journal of Clinical Investigation, vol. 108, no. 9, pp. 1379-1385, 2001.

[34] C. L. Gao, C. Zhu, Y. P. Zhao et al., "Mitochondrial dysfunction is induced by high levels of glucose and free fatty acids in 3T3-L1 adipocytes," Molecular and Cellular Endocrinology, vol. 320, no. 1-2, pp. 25-33, 2010.

[35] T. Wang, Y. Si, O. S. Shirihai et al., "Respiration in adipocytes is inhibited by reactive oxygen species," Obesity, vol. 18, no. 8 , pp. 1493-1502, 2010.

[36] I. Talior, M. Yarkoni, N. Bashan, and H. Eldar-Finkelman, "Increased glucose uptake promotes oxidative stress and PKC-delta activation in adipocytes of obese, insulin-resistant mice," American Journal of Physiology. Endocrinology and Metabolism, vol. 285, no. 2, pp. E295-E302, 2003.

[37] Y. Lin, A. H. Berg, P. Iyengar et al., "The hyperglycemiainduced inflammatory response in adipocytes: the role of reactive oxygen species," The Journal of Biological Chemistry, vol. 280, no. 6, pp. 4617-4626, 2005.

[38] S. Furukawa, T. Fujita, M. Shimabukuro et al., "Increased oxidative stress in obesity and its impact on metabolic syndrome," The Journal of Clinical Investigation, vol. 114, no. 12, pp. 1752-1761, 2004.

[39] N. Houstis, E. D. Rosen, and E. S. Lander, "Reactive oxygen species have a causal role in multiple forms of insulin resistance," Nature, vol. 440, no. 7086, pp. 944-948, 2006.

[40] A. C. Bianco and J. E. Silva, "Intracellular conversion of thyroxine to triiodothyronine is required for the optimal thermogenic function of brown adipose tissue," The Journal of Clinical Investigation, vol. 79, no. 1, pp. 295-300, 1987. 


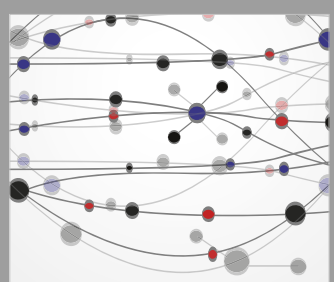

The Scientific World Journal
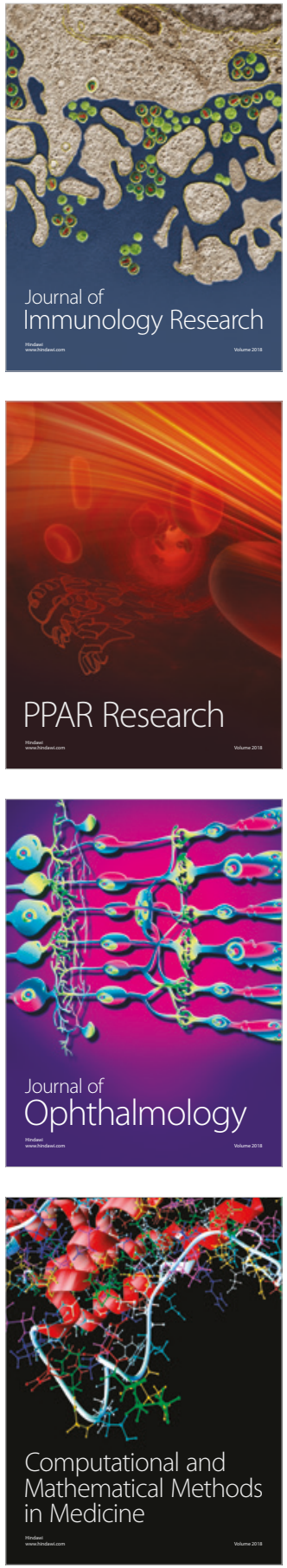

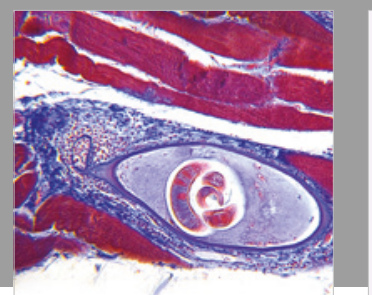

Gastroenterology Research and Practice

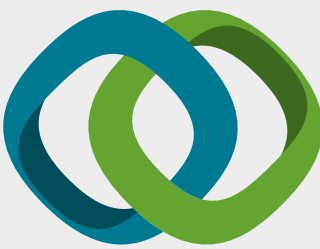

\section{Hindawi}

Submit your manuscripts at

www.hindawi.com
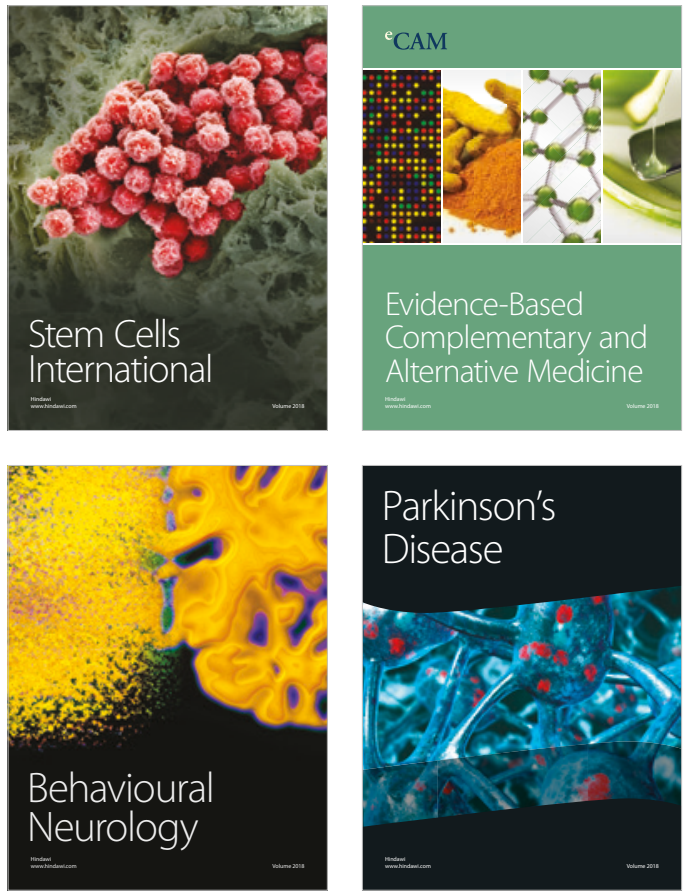

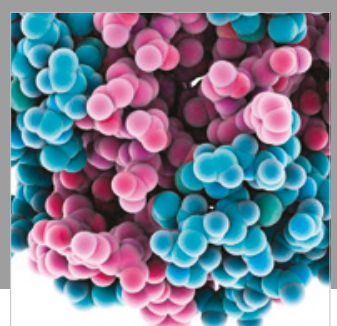

ournal of

Diabetes Research

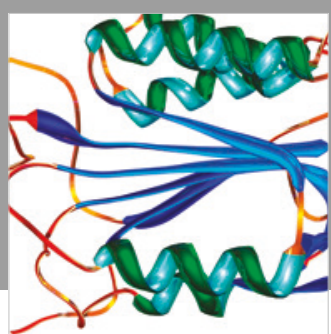

Disease Markers
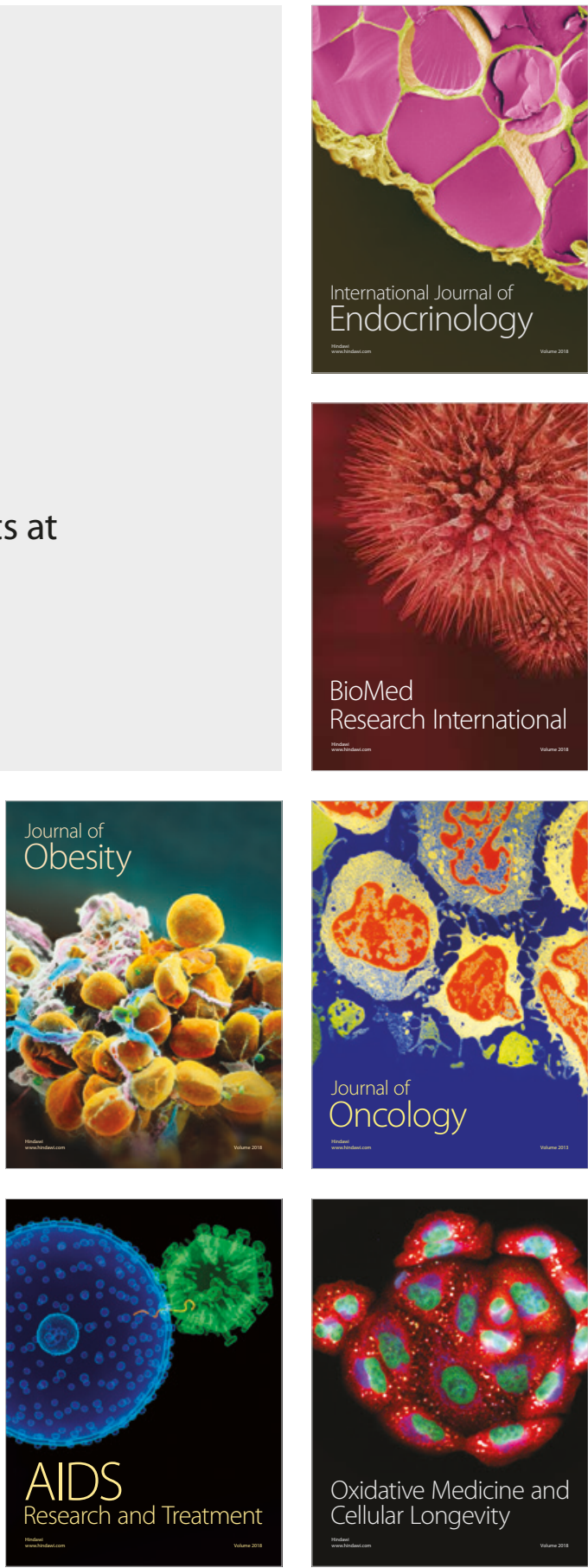